\title{
Simultaneous integrated protection
}

\section{A new concept for high-precision radiation therapy}

\author{
Thomas B. Brunner ${ }^{1,2} \cdot$ Ursula Nestle $^{1,2} \cdot$ Sonja Adebahrr $^{1,2} \cdot$ Eleni Gkika $^{1,2} \cdot$ Rolf Wiehle $^{1,2} \cdot$ Dimos Baltas $^{1,2} \cdot$ \\ Anca-Ligia Grosu ${ }^{1,2}$
}

Received: 10 May 2016 / Accepted: 21 September 2016 / Published online: 18 October 2016 (C) The Author(s) 2016. This article is available at SpringerLink with Open Access.

\begin{abstract}
Objective Stereotactic radiotherapy near serial organs at risk (OAR) requires special caution. A novel intensity-modulated radiotherapy (IMRT) prescription concept termed simultaneous integrated protection (SIP) for quantifiable and comparable dose prescription to targets very close to OAR is described.

Materials and methods An intersection volume of a planning risk volume (PRV) with the total planning target volume (PTV) defined the protection volume $\left(\mathrm{PTV}_{\mathrm{SIP}}\right)$. The remainder of the PTV represented the dominant PTV $\left(\mathrm{PTV}_{\mathrm{dom}}\right)$. Planning was performed using IMRT. Dose was prescribed to $\mathrm{PTV}_{\text {dom }}$ according to ICRU in 3, 5, 8, or 12 fractions. Constraints to OARs were expressed as absolute and as equieffective doses at 2 Gy (EQD2). Dose to the gross risk volume of an OAR was to respect constraints. Violation of constraints to OAR triggered a planning iteration at increased fractionation. Dose to $\mathrm{PTV}_{\text {SIP }}$ was required to be as high as possible within the constraints to avoid local relapse.

Results SIP was applied in 6 patients with OAR being large airways $(n=2)$ or bowel $(n=4)$ in $3,5,8$, and
\end{abstract}

Thomas B. Brunner and Ursula Nestle contributed equally to the manuscript.

Electronic supplementary material The online version of this article (doi: 10.1007/s00066-016-1057-x) contains supplementary material, which is available to authorized users.

Thomas B. Brunner, MD

thomas.brunner@uniklinik-freiburg.de

1 Department of Radiation Oncology, University Hospitals Freiburg, Freiburg, Deutschland

2 Partner Site Freiburg, German Cancer Consortium (DKTK), Heidelberg, Germany
12 fractions in 1, 3, 1, and 1 patients, respectively. PTVs were $14.5-84.9 \mathrm{ml}$ and $\mathrm{PTV}_{\mathrm{SIP}} 1.8-3.9 \mathrm{ml}(2.9-13.4 \%$ of PTV). Safety of the plans was analyzed from the absolute dose-volume histogram (dose to $\mathrm{ml}$ ). The steepness of dose fall-off could be determined by comparing the dose constraints to the PRVs with those to the OARs (Wilcoxon test $p=0.001$ ). Constraints were respected for the corresponding OARs. All patients had local control at a median 9 month follow-up and toxicity was low.

Conclusion SIP results in a median dose of $\geq 100 \%$ to PTV, to achieve high local control and low toxicity. Longer follow-up is required to verify results and a prospective clinical trial is currently testing this new approach in chest and abdomen stereotactic body radiotherapy.

Keywords Stereotactic body radiation therapy · Intensitymodulated radiotherapy $\cdot$ Efficacy $\cdot$ Toxicity $\cdot$ Organs at risk

\section{Simultan integrierte Protektion}

Ein neues Konzept für die Hochpräzisionsbestrahlung

\section{Zusammenfassung}

Zielsetzung Die stereotaktische Radiotherapie nahe serieller Risikoorgane (OAR) erfordert besondere Vorsicht. Wir beschreiben ein neues Konzept für die intensitätsmodulierte Strahlentherapie (IMRT), genannt simultan integrierte Protektion (SIP), das die Dosisverschreibung auf Zielvolumina in unmittelbarer Nähe von OAR quantifiziert.

Material und Methoden Das Überschneidungsvolumen eines Planungsrisikovolumens (PRV) mit dem gesamten Planungszielvolumen (PTV) definierte das Protektionsvolumen $\left(\mathrm{PTV}_{\mathrm{SIP}}\right)$. Der Rest des PTV repräsentierte das dominierende PTV $\left(\mathrm{PTV}_{\mathrm{dom}}\right)$. Die Planung erfolgte mittels 
IMRT. Nach ICRU wurde die Dosis auf PTV dom in 3, 5, 8 oder 12 Fraktionen verschrieben. OAR-Grenzdosen wurden als absolute und äquieffektive Dosen zu 2 Gy (EQD2) überprüft. Die Dosis auf das makroskopische Risikovolumen eines OAR musste die Constraints einhalten. Eine Verletzung der OAR-Constraints triggerte eine Planungswiederholung mit höherer Fraktionszahl. Die PTV SIP-Dosis sollte so hoch wie möglich sein, aber innerhalb der Constraints, um Lokalrezidive zu vermeiden.

Ergebnisse Die SIP-Technik wurde bei 6 Patienten mit den OARs große Atemwege $(n=2)$ und Darm $(n=4)$ in 3, 5, 8 und 12 Fraktionen bei jeweils 1, 3, 1 und 1 Patienten angewendet. PTVs maßen 14,5-84,9 ml und PTV SIP $_{1,8-3,9 ~ m l}$ (2,9-13,4\% des PTV). Die Verträglichkeit der Pläne wurde durch die Analyse der absoluten Dosisvolumenhistogramme (Dosis auf ml) überprüft. Die Steilheit des Dosisabfalls wurde durch den Vergleich der Dosis-Constraints mit der Dosis auf die PRVs gegenüber der Dosis auf die OARs abgelesen (Wilcoxon-Test $p=0,001$ ). Die Constraints für die OARs wurden eingehalten. Alle Patienten zeigten eine Lokalkontrolle bei einem medianen 9-monatigen Nachbeobachtungszeitraum und niedrige Toxizität.

Schlussfolgerung SIP ermöglichte eine mediane Dosis von $\geq 100 \%$ auf das PTV und ergab eine hervorragende Lokalkontrolle bei niedriger Toxizität. Die Verifikation der Ergebnisse erfordert eine längere Nachbeobachtungszeit. Eine prospektive klinische Studie testet derzeit diesen neuen Ansatz für die thorakale und abdominelle Körperstereotaxie.

Schlüsselwörter Stereotaktische Körperbestrahlung · Intensitätsmodulierte Strahlentherapie · Effizienz ·

Toxizität · Risikoorgane

\section{Introduction}

Over the past two decades stereotactic radiotherapy (SRT) has evolved to a powerful tool to control lesions especially in the brain, lungs, and liver [11, 12, 19, 21, 23]. However, reports of high-grade toxicities after stereotactic body radiotherapy (SBRT) of central lung tumors and of lesions near the bowel or stomach on the other hand demonstrated the difficulties to safely administer SBRT in these situations despite multimodal imaging, accurate motion management, intensity-modulated radiotherapy (IMRT), and image-guided radiotherapy (IGRT) [9, 22, 26].

The concept of SRT relies on avoiding organs at risk (OARs) through high spatial precision. Inherently, limitations of SRT and SBRT were encountered when the target lesions were too close to OARs and this is the clinical problem stipulating the development of the currently described novel concept of simultaneous integrated protection (SIP). For example, perforation of central airways, bronchial hem- orrhage, perforations of the esophagus, stomach, or small bowel were observed [7, 14, 26]. Strategies to reduce the risk of high-grade toxicities often rely on the prescription of reduced total dose to the entire planning target volume (PTV). However, reduction of the total dose comes at the price of lowered local tumor control $[8,20]$. Another strategy is to increase the number of fractions to exploit the differential radiosensitivity of tumor and OARs as defined by their $\alpha / \beta$ ratios $[1,3]$. While this will help to overcome some of the limitations in more critical locations, there are still a significant number of cases where neither reduction of the total dose nor increasing the number of fractions within the limits of significant hypofractionation enables the application of an adequate radiation dose. The above mentioned clinical reports of high-grade and even fatal complications after SBRT illustrate the consequences of nonadherence to these rules $[14,26]$. The tradition of prescribing SBRT not according to ICRU but to individually chosen isodoses (typically 60-80\%) aggravates the problem due to steep dose gradients which make even small setup errors highly risky. In summary, there is no standard approach to overcome such obstacles of the safe application of SBRT at this time.

When change of dose or fractionation is not sufficient, this problem is often addressed by individual dose reductions at the interface of target lesions with a critical organ at risk (OAR) at the discretion of the treating physician. However, in addition to the lack of data in the literature, such compromises derived from the fear of normal tissue complications may lead to the application of insufficient tumor doses and impair local control [20]. Furthermore, the lack of interobserver and interinstitutional comparability is a cardinal factor of inconsistencies, and it is a problem for prospective trials.

Our aim was therefore to develop a prescription method maximizing consistency of SBRT hypofractionation plans for targets near OARs [5] which deliberately and in a calculated way lowers the dose to a part of the PTV that is close to critical OARs. We present a method named simultaneous integrated protection (SIP) in analogy to the simultaneous integrated boost (SIB) intensity-modulated radiotherapy (IMRT) technique. It is based on the definition of a subvolume being the intersection of the PTV and the PRV of a critical OAR to which the highest possible dose respecting the dose constraints for this OAR is planned and delivered.

\section{Materials and methods}

The stepwise procedure to define the SIP approach is summarized in Table 1, while a flowchart is shown in Supplemental Fig. 1. The SIP concept was required for SBRT 
Table 1 Standardized definition of the simultaneous integrated protection (SIP) concept in the treatment planning algorithm

\begin{tabular}{|c|c|}
\hline $\begin{array}{l}\text { 1. Indication: The use of the technique is } \\
\text { indicated when }\end{array}$ & $\begin{array}{l}\text { - First, the standard indications for stereotactic radiotherapy are given (not further specified in the } \\
\text { framework of this manuscript) and } \\
\text { - Second, there is overlap of the planning target volume (PTV) with a critical organ at risk (OAR) } \\
\text { or with the planning risk volume (PRV) of this OAR }\end{array}$ \\
\hline 2. Definitions of volumes (Fig. 1): & 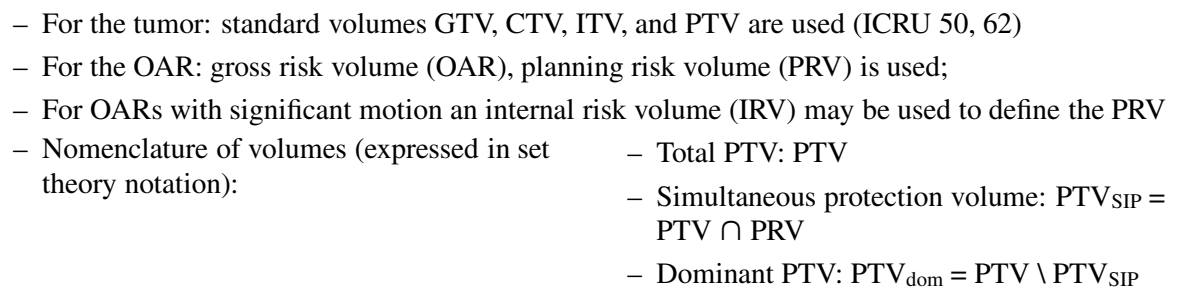 \\
\hline 3. Prescription: & 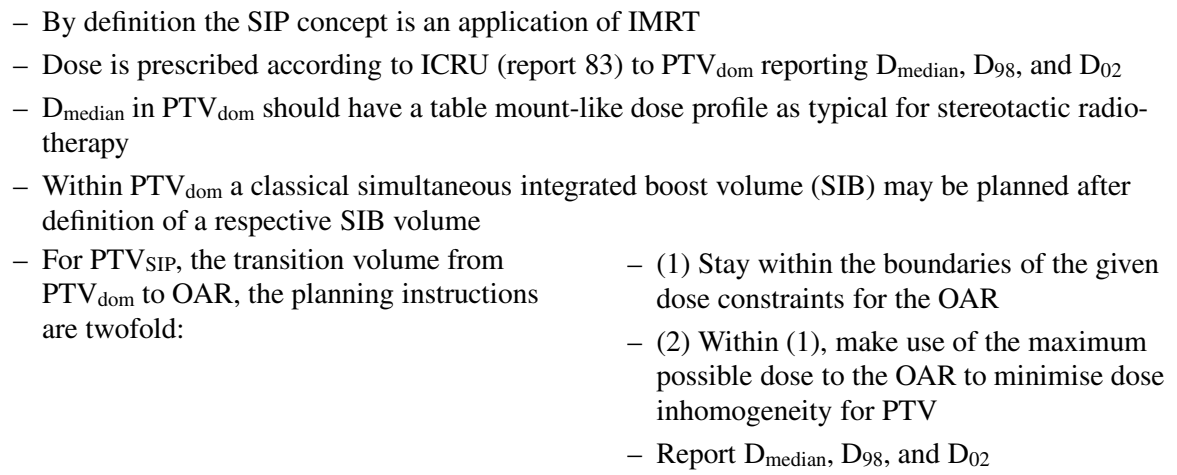 \\
\hline
\end{tabular}

4. Dose constraints for OARs: dose constraints as published by QUANTEC or other are employed in biologically equivalent form, e. g., as EQD2 calculated with the appropriate $\alpha / \beta$ values. These need to be at the highest level of evidence available and have to be updated accordingly

5. Analysis:

- Individual RTQA is performed if all boundaries are met at plan analysis for the PTV $\mathrm{dom}_{\text {and }}$ for the OAR

- If constraints are violated:

- Planning iterations with a higher number of fractions up to a specified maximum (e. g., 12 fractions) are performed

- Here, prescribed doses and dose constraints are recalculated to the equieffective dose at 2 Gy (EQD2) using the respective $\alpha / \beta$ values of tumor and OAR aiming to deliver the maximum possible EQD2 to the tumor with acceptable toxicity

- If the limits of the normal tissue constraints cannot be kept hereby, SBRT should not be given but rather conventionally fractionated treatment

6. IGRT: cutting edge patient positioning and IGRT is mandatory for the use of the SIP concept

treatment planning in case where there was an unacceptable high dose to an OAR, i. e., if there was overlap of the PTV with either the OAR or its expansions (IRV, PRV). This represented the key inclusion criterion for this report. Patients with an indication for SBRT where the SIP concept did not achieve adequate protection of the OAR at the highest planned fraction number, i.e., 12 fractions, were not eligible as described below.

This approach requires accurate definitions of the volumes to be treated. For the tumor, these were defined as GTV, CTV, ITV, and PTV in accordance with the ICRU reports 50 and 62 . For the OAR, we used terms that are analogous with those for the tumors, i.e., OAR, IRV, and
PRV. We defined the OAR as the volume segmented in the planning CT, and the IRV as the volume derived from 4DCT. Describing the relation of PTV and the PRV to each other (Fig. 1), we defined the nomenclature of volumes in set theory notation to be 1) PTV for the total PTV, 2) the simultaneous protection volume $\left(\mathrm{PTV}_{\mathrm{SIP}}\right)$ for the intersection of the PTV and the PRV, PTV ${ }_{\text {SIP }}=\mathrm{PTV} \cap \mathrm{PRV}$, and 3) the PTV without intersection with the PRV as the dominant PTV, PTV $_{\text {dom }}=$ PTV $\backslash$ PTV $V_{\text {SIP. }}$ The term dominant was chosen to imply that the SIP approach is only valid for small volumes of PTV $\mathrm{SIP}_{\text {. }}$

Third, we defined the prescription of dose to the above volumes. The concept inherently requires an IMRT ap- 


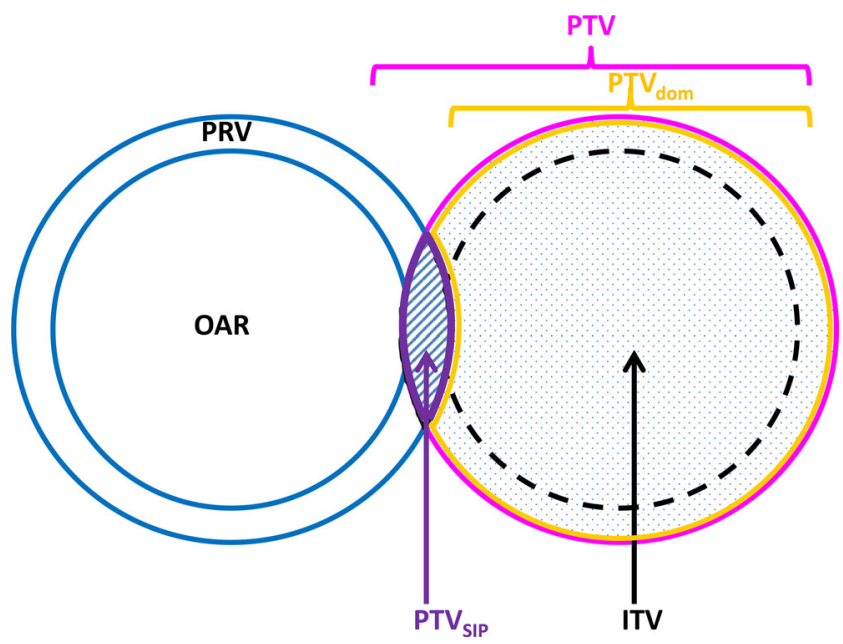

Fig. 1 Contouring and planning using the simultaneous integrated protection (SIP) concept. Scheme of a critical organ at risk (OAR; blue, left side) with its planning risk volume $(P R V)$ overlapping with the planning target volume $(P T V$, pink $)$. The dominant $\mathrm{PTV}\left(\mathrm{PTV}_{\mathrm{dom}}=\right.$ PTV $>P R V$; orange) is the prescribed dose in the conventional way,

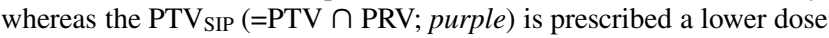
to stay within the dose constraints for the OAR

proach for simultaneous administration of different doses to PTV $_{\text {dom }}$ and to PTV $\mathrm{PIP}_{\text {. }}$. Dose was prescribed according to ICRU report 83 to $P T V_{\text {dom }}$ indicating $\mathrm{D}_{\text {median }}, \mathrm{D}_{98}$, and $\mathrm{D}_{02}$. As typical for SBRT, we were asking for a table mountlike dose profile in $\mathrm{PTV}_{\text {dom }}$ and a $\mathrm{D}_{02}$ being up to $120 \%$ of the prescribed dose [25]. In addition, the SIP concept was combined with a simultaneous integrated boost (SIB) in some of the patients. For PTV $\mathrm{PIP}_{\mathrm{SI}}$, i. e., the volume that contains the dose gradient from $\operatorname{PTV}_{\text {dom }}$ to the $\operatorname{OAR}(\mathrm{s})$, the planning instructions were twofold:

- to stay within the boundaries of the given dose constraints for the OAR itself, and

- to make use of the maximum possible dose to PTV $\mathrm{PIP}_{\text {SI }}$ to minimize dose inhomogeneity for PTV.

In order to ensure this, the dose gradient between the dose to the OAR and the dose prescribed to the PTV $\mathrm{V}_{\mathrm{dom}}$ typically localizes to the PRV volume around the OAR. We also reported $\mathrm{D}_{\text {median, }} \mathrm{D}_{98}$, and $\mathrm{D}_{02}$ for PTV $\mathrm{PIP}_{\text {SIP }}$ to quantify the dose sacrifice that was made for the PTV of a distinct lesion.

Fourth, we carefully chose available dose constraints for the OARs following the recommendations published by QUANTEC and other published recommendations commonly used for SBRT $[1,5,12,16,27,28]$. For the respective fractionation schedules, dose constraints for OAR were calculated as equieffective doses in 2 Gy fractions (EQD2) with corresponding $\alpha / \beta$ ratios $(\alpha / \beta 3$ for large airways, bowel structures).

Fifth, plans were checked for all boundaries as defined above prior to individual RTQA (Fig. 2). In cases where the dose constraints were violated at a chosen number of fractions, planning iterations with a higher number of fractions up to a specified maximum of 12 fractions were performed. Prescribed doses and dose constraints were recalculated to the EQD2 using the respective $\alpha / \beta$ ratios of tumor and OAR, and aiming to deliver iso-effective doses to the tumor with lower toxicity by protracted dose delivery. If the normal tissue constraints could not be fulfilled by increasing the number of fractions to the maximum number, SBRT was not given but conventionally fractionated treatment performed instead. Sixth, we excluded lesions with

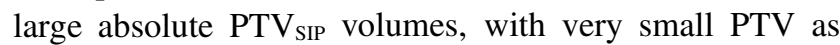
well as for single fraction radiosurgery from the use of the SIP concept. Seventh, we required high-precision patient positioning, motion management, and IGRT for the use of the SIP concept.

For the analysis descriptive statistics were used and the Wilcoxon test for paired differences was used for the comparison of dose to the PRVs with dose to the respective OARs to evaluate the plans for given dose constraints. Kaplan-Meier statistics were employed to calculate local control after therapy.

\section{Results}

In this article, we describe the clinical application of the SIP concept. Six patients with indications for SBRT of targets close to OAR underwent 4D treatment planning imaging with high-precision positioning. Two had lesions in the chest, one in the liver, two in the pancreas and one in the left kidney. One patient (\# 3) was treated with a non-SIP SBRT plan with reduced dose (5 fractions of 6 Gy to $60 \%$ isodose of ITV) for a central lung metastasis close to the right hilum but had an in silico SIP plan to full dose for PTV $_{\text {dom. }}$ All other patients were treated with the SIP plan. The size of the PTVs (PTV) ranged from 14.5-84.9 ml (median $49.15 \mathrm{ml}$, mean $49.57 \mathrm{ml}$; Fig. 3). Sizes of PTV protection subvolumes $\left(\mathrm{PTV}_{\mathrm{SIP}}\right)$ ranged from $1.0-3.9 \mathrm{ml}$ (median 2.65 and mean $2.60 \mathrm{ml}$ ). Relative $\mathrm{PTV}_{\text {SIP }}$ ranged from $2.9-13.4 \%$ of the size of PTV (median $5.9 \%$ ). Noteworthy, the largest ratio, $13.4 \%$, was an absolute volume

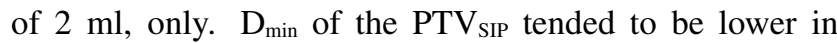
patients 1,2 , and 6 due to air within the PTV $\mathrm{SIP}_{\text {Solumes }}$ compared with the other patients. Safety of the plans was analyzed from the absolute volume DVHs as summarized in Supplemental Table 1 showing the comparison of the dose constraints with the doses in the plans to the OARs of the OARs and to the PRVs. The steepness of dose fall off can be read off by the comparing the doses to the PRVs with those to the OARs. Expectedly, the dose constraints for the respective OARs in the PRV volumes were violated for some of the PRVs but the constraints were respected for the 

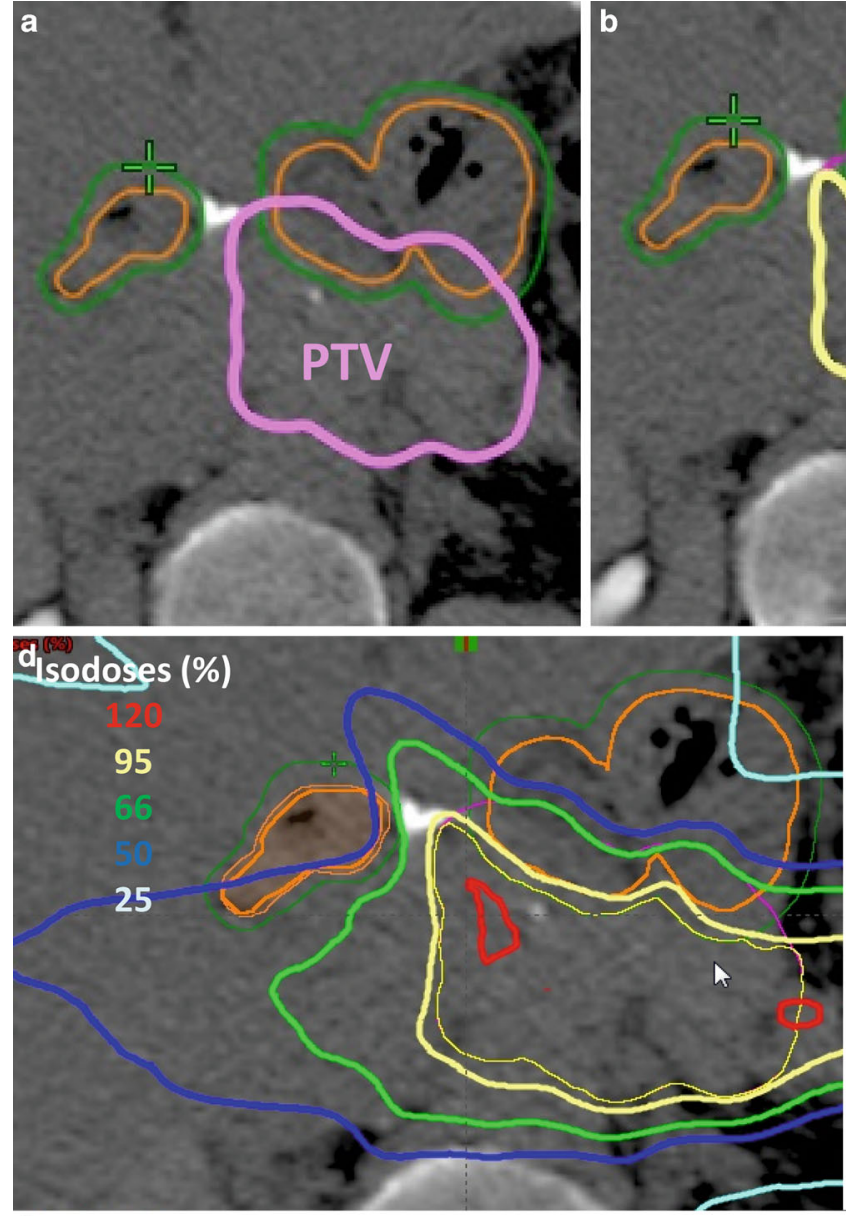

f

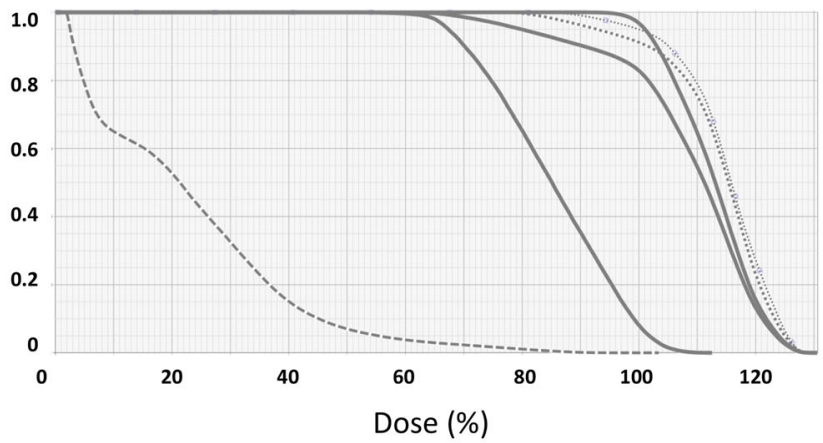

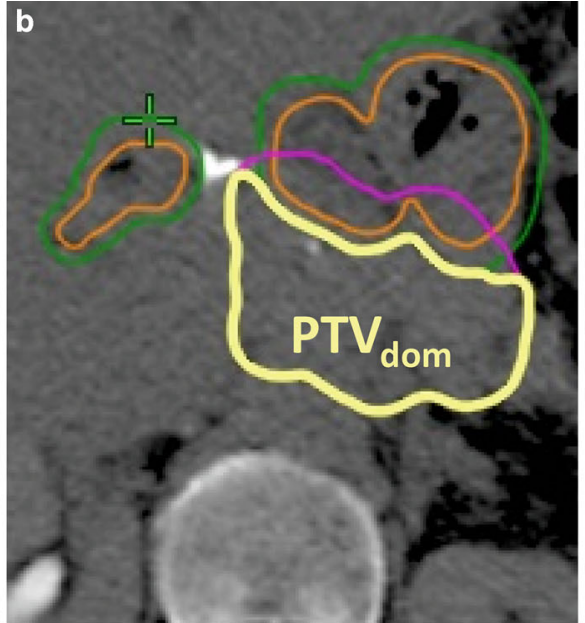
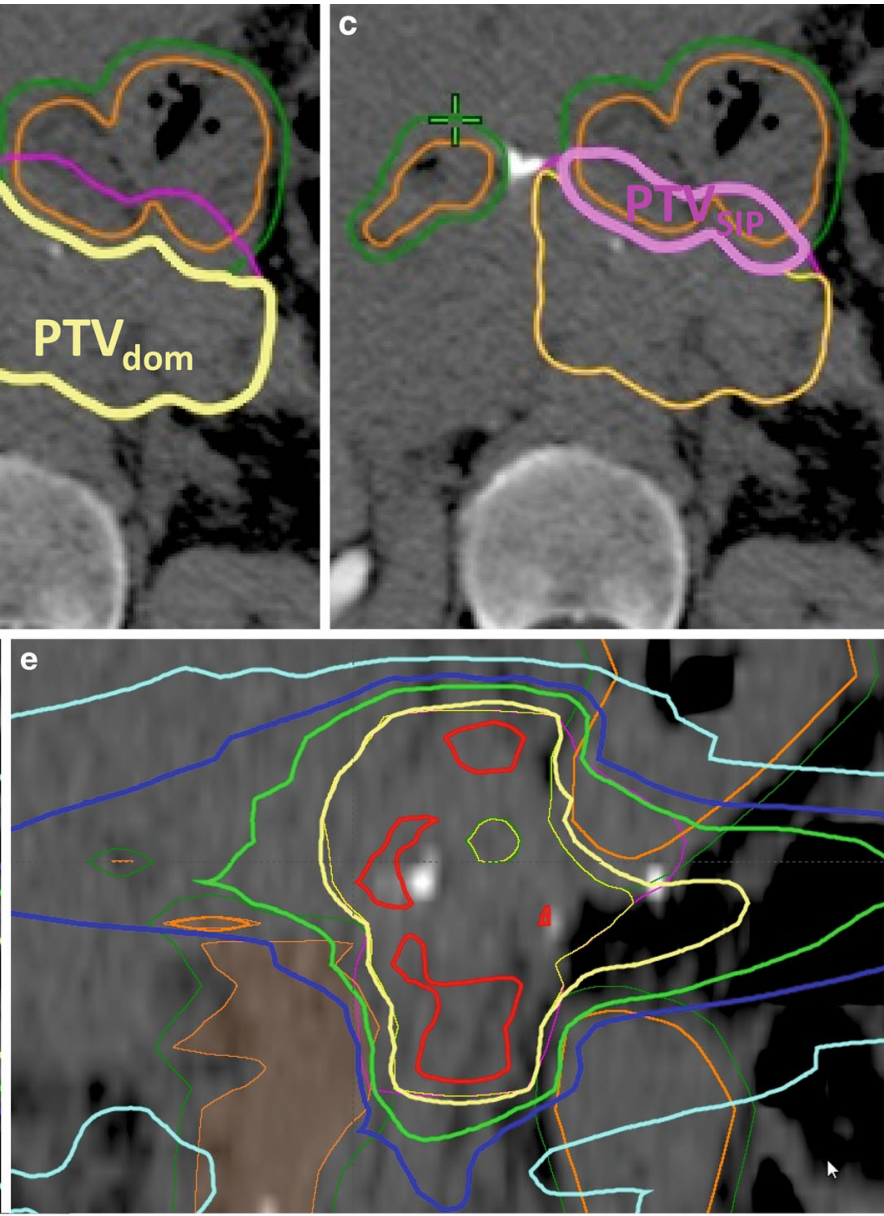

g

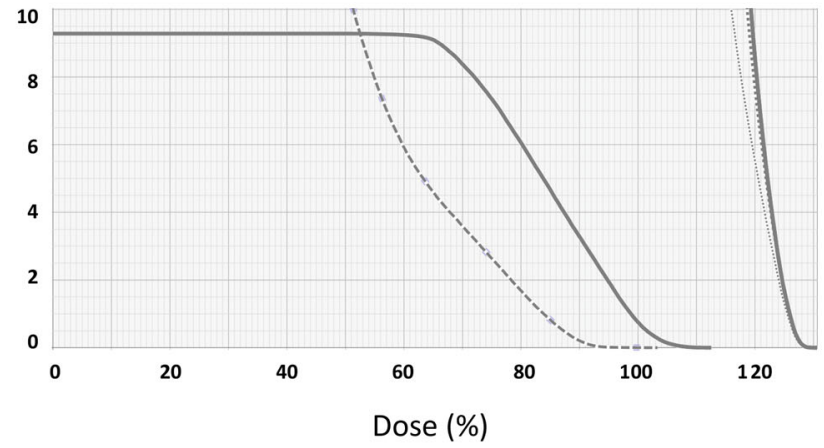

Fig. 2 a The planning target volume ( $P T V$, (light pink) intersects with planning risk volume (PRV, green) and organs at risk (OAR), small bowel (orange), in a patient with recurrent pancreatic cancer after resection. b The PRV is subtracted from PTV to define PTV dom $_{\text {yellow). }} \mathbf{c}$ The $\mathrm{PTV}_{\mathrm{SIP}}$ is defined as the intersecting region of PTV with the PRV (PTV $\cap$ PRV; magenta). d,e An intensity-modulated radiotherapy (IMRT) plan is developed to deliver full dose according to ICRU to PTV dom $_{\text {and a lower dose to PTV }}$ SIP respecting the dose constraints for small bowel in 12 fractions $\left(\mathrm{D}_{\max }=47.4 \mathrm{~Gy}, \mathrm{D}_{0.5 \mathrm{ml}}=44.5 \mathrm{~Gy}, \mathrm{D}_{5 \mathrm{ml}}=44.4 \mathrm{~Gy}\right)$. Isodose levels as stated on the left side. $\mathbf{f}$ Relative dose-volume histogram $(D V H)$.

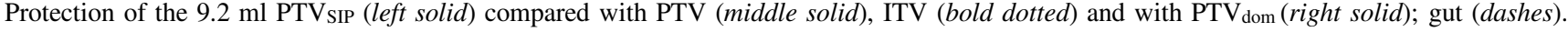
g Absolute DVH respecting the constraints for gut

corresponding OARs as an indirect sign for the successful application of the SIP concept (Supplemental Table 1). To quantify this, a comparison of the given dose constraints with the actual doses to the OAR volumes and the PRV volumes was performed by analyzing the difference of the ratio D (OAR)/D (constraint) with D (PRV)/D (constraint) for the 19 dose constraint values shown in Supplemental Table 1 ( $p=0.001$, Wilcoxon test). None of these patients showed severe toxicity within a median follow-up of 8.6 months (range 3.1-26.2 months) with favorable local control $(100 \%)$. 
Fig. 3 Dose to planning target volumes $(P T V)$ in 6 patients planned with the simultaneous integrated protection $(S I P)$ concept. For each patient from left to right the relative prescription doses to the dominant non-protection PTV, PTV, and PTV SIP are shown with minimal (min), maximal (max), and mean (filled diamonds) relative doses. OAR organ at risk

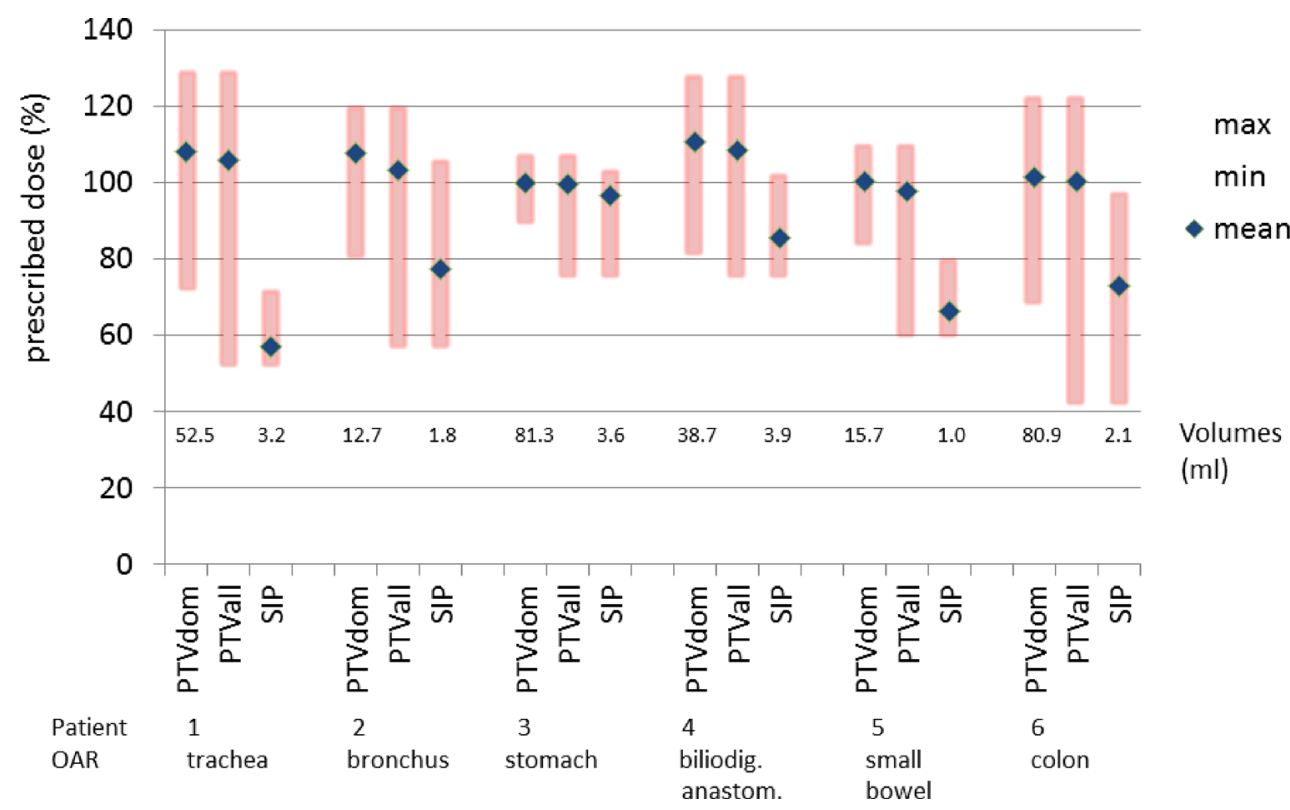

Table 2 Relative dose parameters for all planning target volume (PTV) types in 6 patients

\begin{tabular}{|c|c|c|c|c|c|c|c|c|c|c|}
\hline Patient & Target lesion & PTV type & $D_{\text {mean }}$ & $\mathrm{D}_{\min }$ & $\mathrm{D}_{\max }$ & $\mathrm{D}_{98}(\%)$ & $\mathrm{D}_{95}(\%)$ & $\mathrm{D}_{02}(\%)$ & $\mathrm{V}_{95}(\%)$ & $\mathrm{V}_{107}(\%)$ \\
\hline \multirow[t]{3}{*}{$\overline{1}$} & \multirow[t]{3}{*}{ M_pul (CRC) } & PTV & 106.0 & 52.7 & 128.3 & 66.7 & 77.1 & 125.3 & 81.8 & 54.68 \\
\hline & & $\mathrm{PTV}_{\mathrm{dom}}$ & 108.19 & 72.7 & 128.3 & 85.9 & 89.8 & 125.4 & 86.96 & 58.09 \\
\hline & & $\mathrm{PTV}_{\mathrm{SIP}}$ & 70 & 52.7 & 86.5 & 56.3 & 58.5 & 81.2 & 0 & 0 \\
\hline \multirow[t]{3}{*}{2} & \multirow[t]{3}{*}{ NSCLC } & PTV & 110.2 & 60.7 & 125.4 & 70.2 & 73.3 & 122.8 & 87.4 & 79.1 \\
\hline & & $\mathrm{PTV}_{\mathrm{dom}}$ & 115.2 & 87.1 & 125.4 & 101.8 & 105.7 & 123 & 99.8 & 92.7 \\
\hline & & $\mathrm{PTV}_{\text {SIP }}$ & 79.5 & 61.6 & 110.4 & 67.7 & 68.9 & 100.6 & 8.7 & 0.03 \\
\hline \multirow[t]{3}{*}{3} & \multirow[t]{3}{*}{$\mathrm{HCC}$} & PTV & 99.5 & 67.3 & 106.1 & 89.7 & 95.8 & 103.8 & 95.96 & 0 \\
\hline & & $\mathrm{PTV}_{\mathrm{dom}}$ & 100 & 90 & 106.1 & 96.5 & 97.8 & 102.7 & 99.4 & 0 \\
\hline & & $\mathrm{PTV}_{\mathrm{SIP}}$ & 67.3 & 75.9 & 102.3 & 85.5 & 88.9 & 101.0 & 75.56 & 0 \\
\hline \multirow[t]{3}{*}{4} & \multirow[t]{3}{*}{ LR-PDAC } & PTV & 108.4 & 75.9 & 126.9 & 94.1 & 96.5 & 124.7 & 97.02 & 60.65 \\
\hline & & $\mathrm{PTV}_{\mathrm{dom}}$ & 110.8 & 81.7 & 126.9 & 97.5 & 101.6 & 124.8 & 99.18 & 67.02 \\
\hline & & $\mathrm{PTV}_{\text {SIP }}$ & 85.5 & 75.9 & 101.2 & 81.9 & 85.8 & 94.6 & 88.06 & 0 \\
\hline \multirow[t]{3}{*}{5} & \multirow[t]{3}{*}{ LR-PDAC } & PTV & 97.8 & 60.4 & 108.9 & 65.1 & 69.3 & 105.0 & 84.07 & 0.11 \\
\hline & & $\mathrm{PTV}_{\mathrm{dom}}$ & 100.5 & 84.4 & 108.9 & 92.7 & 93.4 & 105.1 & 93.33 & 0.12 \\
\hline & & $\mathrm{PTV}_{\mathrm{SIP}}$ & 66.4 & 60.4 & 78.6 & 62.1 & 62.6 & 74.8 & 0 & 0 \\
\hline \multirow[t]{3}{*}{6} & \multirow[t]{3}{*}{ Renal cancer } & PTV & 120.6 & 51.5 & 145.6 & 91.5 & 94.8 & 143.0 & 94.85 & 78.16 \\
\hline & & $\mathrm{PTV}_{\mathrm{dom}}$ & 121.6 & 82.7 & 145.6 & 93.4 & 96.3 & 143.1 & 96.43 & 80.35 \\
\hline & & $\mathrm{PTV}_{\text {SIP }}$ & 87.7 & 51.5 & 115.1 & 57.2 & 61.0 & 109.3 & 39.6 & 5.04 \\
\hline
\end{tabular}

Maximal doses were prescribed either with a table-mount profile (patients 1, 2, 4, 6) or without (patients 3, 5)

$H C C$ hepatocellular carcinoma, LR-PDAC locally relapsed pancreatic cancer, $M_{-}$pul $(C R C)$ lung metastasis from colorectal cancer, $N S C L C$ non-small cell lung cancer

On the other hand, the dose trade-off to the PTVs due to SIP was also quantified. Mean doses to the PTVs were compared between the three volumes $\left(\mathrm{PTV}, \mathrm{PTV}_{\mathrm{dom}}\right.$, $\left.\mathrm{PTV}_{\mathrm{SIP}}\right)$ as shown in Table 2. Comparing $\mathrm{D}_{\text {mean, PTV }}$ with $\mathrm{D}_{\text {mean }}, \mathrm{PTV}_{\text {dom }}$, the difference was just about significant at $p=0.043$ whereas the difference was more significant between $\mathrm{D}_{\text {mean, PTV }}$ with $\mathrm{D}_{\text {mean, }} \mathrm{PTV}_{\mathrm{SIP}}$ at $p=0.028$ (Wilcoxon test). Mean and median relative doses to $95 \%$ (D95 vol.$\%$ ) of the volumes PTV ${ }_{\text {dom }}$, PTV, and PTV SIP $_{\text {were }} 122$,
105 , and $90 \%$, as well as 120,107 , and $93 \%$, respectively. This reflects that the dose sacrifice to $\mathrm{PTV}_{\text {SIP }}$ was kept to a minimum. Maximum BED $(\alpha / \beta$ 10) doses in the PTV for the 6 patients were 124, 135, 93, 92, 114, and $154 \mathrm{~Gy}$, respectively. Fig. 4 and Supplemental Table 2 show further examples of applications of the SIP concept for conventionally fractionated IMRT for cerebral and extracerebral target volumes. 

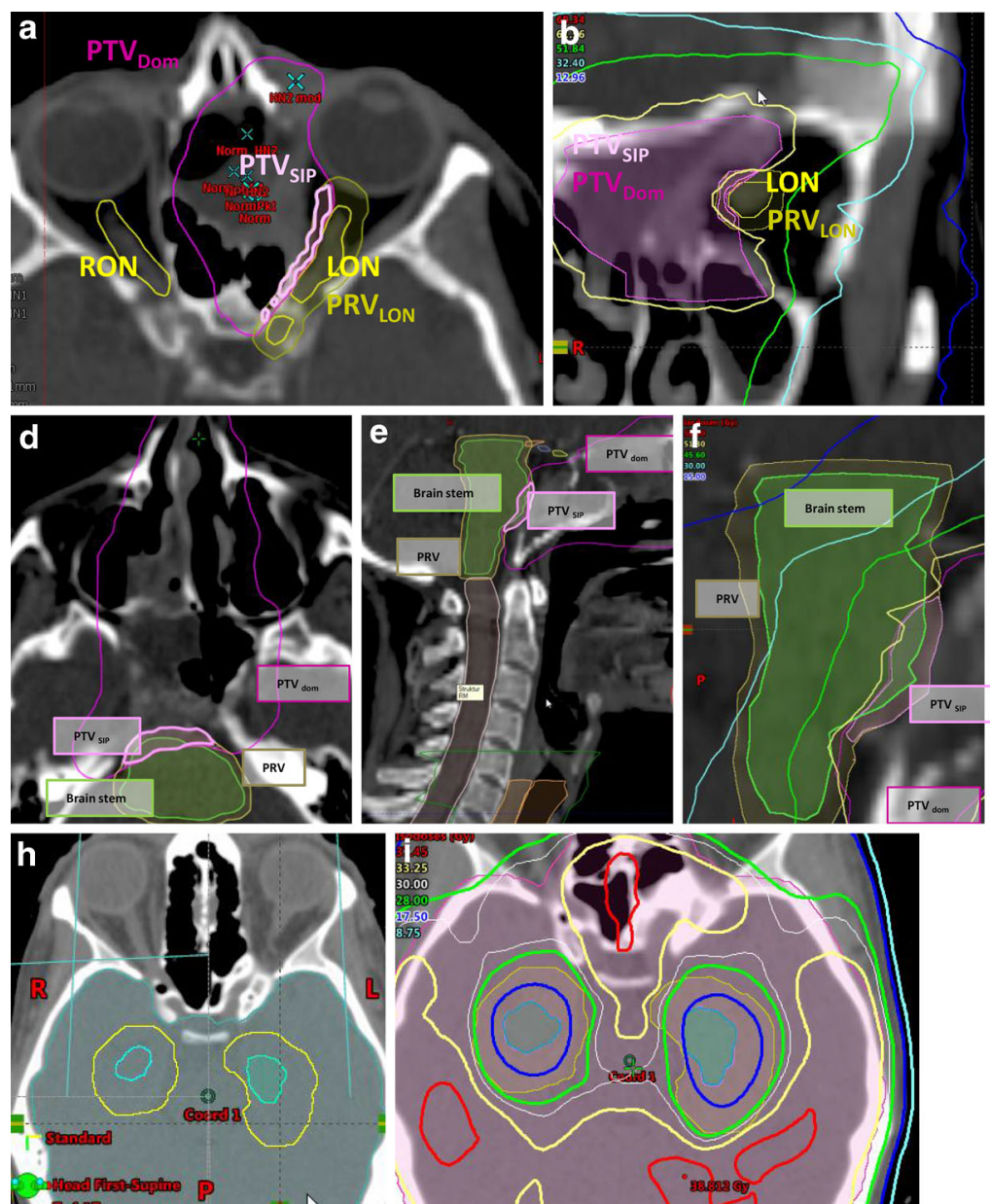
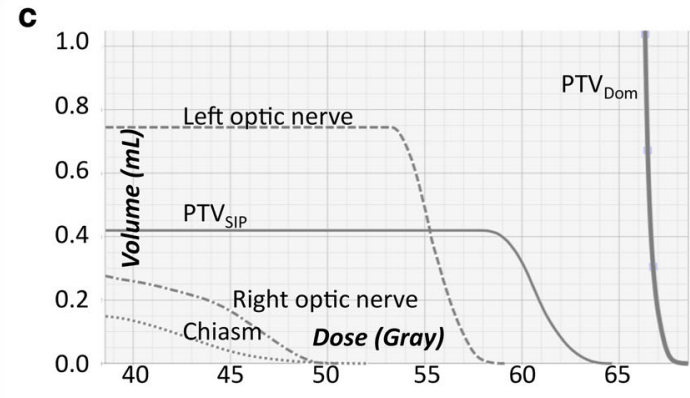

g

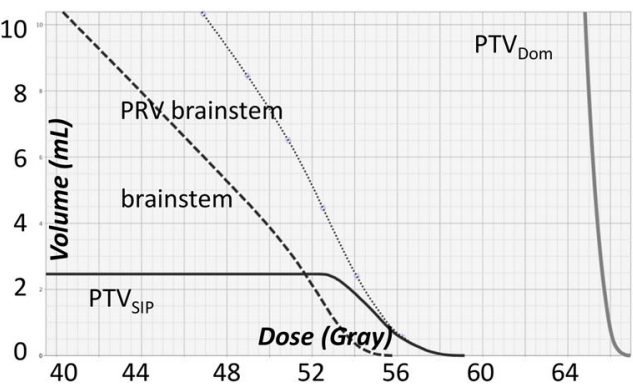

j

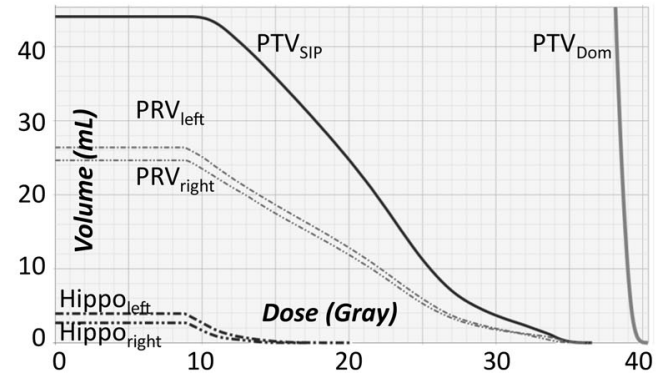

Fig. 4 Examples for a simultaneous integrated protection (SIP) for the optic nerve, the brainstem, and the brachial plexus with dose parameters in Supplementary Table 2. a Axial planning CT of a patient with sinonasal squamous cell carcinoma who was treated with chemoradiotherapy after

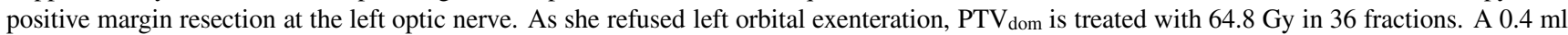
SIP volume is employed to respect a $60 \mathrm{~Gy}$ constraint to the left optic nerve. b The coronal plane visualizes the yellow 61.5 Gy isodose line around the nerve. c Absolute dose-volume histogram $(D V H)$. d Axial and e sagittal planning CT of a patient with undifferentiated main nasal cavity carcinoma with initially direct contact to the brainstem which was shifted dorsally. Tumor shrinkage after two courses of induction chemotherapy with paclitaxel/cisplatin, then chemoradiotherapy with SIP-IMRT to 54 Gy during phase 1 followed by an adaptive sequential boost (not shown) during phase 2. f Isodoses at the interface between the PTV and the brainstem. $\mathbf{g}$ The brainstem constraint of 53 Gy is met as shown in the absolute DVH. h-j Hippocampus protection. $\mathbf{h}$ Delineation of the right (sky blue) and left (blue-green) hippocampus with the respective PRVs (yellow) that

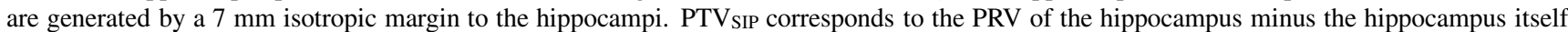
$\left(\mathrm{PTV}_{\text {SIP }}=\mathrm{PRV}_{\text {[side] }} \backslash\right.$ hippocampus[side] $)$. i A total dose of $35 \mathrm{~Gy}$ in 14 fractions was prescribed to the PTV $\mathrm{V}_{\text {dom. Note }}$ the $28.0 \mathrm{~Gy}$ (green) and the $17.5 \mathrm{~Gy}$ (cornflower blue) isodoses at the two SIP volumes. $\mathbf{j}$ In the absolute DVH, the hippocampi receive a mean dose of $\leq 10 \mathrm{~Gy}$. PRV planning risk volume, $P T V$ planning target volume

\section{Discussion}

The described technique of the SIP concept proposes a fully quantified method to protect OARs and to avoid toxicity in a deliberate and reproducible way, while keeping the dose to the remaining PTV at effective levels. The main advantage of this approach is the high level of transparency which makes it a suitable tool for multicenter trials in SBRT minimizing interinstitutional technical differences as a source of error. However, the concept is not restricted to SBRT but could also be used for conventional IMRT or even brachytherapy. In contrast to the SIB method where a small subvolume inside a PTV is prescribed to receive an escalated dose to enhance local control, the SIP concept prescribes a lower dose to a subvolume of a PTV with a high risk of severe toxicity.

The SIP concept is proposed for serial OARs according to the model of functional subunits (FSU) [29]. For serial organs, e.g., spinal cord, esophagus, and bowel, the defect of a few FSUs has a high likelihood for toxicity compared to parallel OARs such as the lung or the liver.

Using the SIP concept, the protection of an OAR is achieved by a protective outer shell around an OAR as a volume for the steep dose gradient between the tumor 
and the OAR. It is important to be aware that the definition of the protection volume of an OAR depends critically on its nature, e.g., PTV $\mathrm{PIP}_{\mathrm{P}}$ can be smaller to protect the chiasm compared to the stomach due to motion. For lesions in the chest, respiratory movements of OARs are of specific importance. Correspondingly, peristalsis of the gut is important in the abdomen [24]. Oral contrast prior to each fraction is recommended in upper abdominal SBRT for IGRT to visualize day-to-day changes. Summarizing, IGRT is key to verify whether employed margins of OARs are correct and clinical trials will have to verify whether the concept is useful and whether the dose constraints were correct. Adaptive radiotherapy strategies can also be combined with the SIP concept and we are currently analyzing this approach in prostate cancer IMRT for the rectum. However, we felt that this would be too complex in the framework of this article and therefore we plan to describe this in a subsequent publication.

Excessive contact volumes between the tumor and the OAR are not thought to be a good indication for SIP. It is

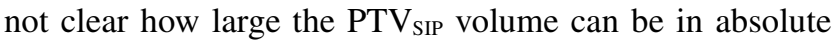
and relative values without a significant loss of tumor control. However, reporting $\mathrm{D}_{\text {median }}, \mathrm{D}_{98}$, and $\mathrm{D}_{02}$ for all target volumes can help to recognize the limits of SIP. Meticulous DVH analysis for target volumes and OARs alike is necessary, but currently, we cannot quantify the risk of local recurrence with SIP. Therefore, prospective trials have to evaluate local relapse and toxicity. The bystander effect may support local tumor control [18]. But it is advisable to exhaust the dose constraints to avoid the risk of local relapse. At this time it is not fully clear yet which dose parameters are most important for local tumor control, PTV encompassing dose, or maximum dose [15]. In a recent analysis of more than 1500 SBRT treatments of primary and secondary tumors of the lung with a broad range of primaries, a plateau of the dose-response curve with $90 \%$ local tumor control probability was reached at $160 \mathrm{~Gy}$ BED when using the PTV maximum dose [13]. Their report supports the view to aim to a give a high dose to large parts of the PTV with a maximum dose at the center of the PTV where the likelihood of tumor location of a moving target is highest. If this concept is correct, then it might be possible to use the SIP concept with a lower dose in a peripheral subvolume of the PTV with lower likelihood of tumor cells being present without compromising local control. In this context it is also intriguing that in parotid sparing head and neck IMRT locoregional recurrences were not observed to occur predominantly in the spared areas but within the highdose regions $[1,25]$. The safety of SIP critically depends on the reliability of the chosen dose constraints which also need to be validated in prospective trials.

From the point of view of radiation biology, it should be stressed that the tumor front might harbor especially ra- dioresistant subvolumes of the tumor. Such a pattern was described in rectal cancer after neoadjuvant therapy and resection $[4,10]$. Epithelial mesenchymal transition (EMT) was described to be more prevalent in residual tumor subvolumes at the invasion front [4] which in turn was described to be enriched for cancer stem cells [2]. Another important factor of resistance to radiotherapy is hypoxia which is not restricted to the tumor core but also is found in subvolumes of the invasive front again warning from low doses at the edge of the tumor [6]. At the moment we cannot adequately image regions of hypoxia, stemness, and EMT in patients and therefore the dose sacrifices should always be as small as possible and this is a hallmark of the here described SIP technique.

In cases where the dose constraints are violated by very hypofractionated approaches (e. g., 3 or 5 fractions), more fractions can reduce the EQD2 for late toxicity due to low $\alpha / \beta$ values. Therefore, we use SIP up to $\leq 12$ fractions for targets with intensive contact to OARs [17]. With the EQD2 formula, isoeffective and isotoxic fractionations should be calculated.

The prescription technique for SBRT described here allows accurate quantification of the dose delivered to dose limiting OARs based on the SIP approach. This system has two advantages: The dose sacrifice to the PTV due to the proximity to a dose-limiting OAR is fully quantified and can be used for local control analysis. At the same time the dose delivered to OARs and to PRVs of OARs can likewise be accurately quantified and therefore be used for toxicity evaluations. This method can be used for SBRT with all SBRT equipment and is suitable for multicenter trials.

\section{Conclusion}

We present a concept for SBRT and IMRT close to highrisk OARs that is expected to be safe and effective and at the same time suitable for multicenter clinical testing. We currently test this approach in a single center phase I trial in patients with thoracic and abdominal lesions and we are confident to thereby further increase the safety of SBRT.

\section{Compliance with ethical guidelines}

Conflict of interest T.B. Brunner, U. Nestle, S. Adebahr, E. Gkika, R. Wiehle, D. Baltas, and A.-L. Grosu declare that they have no competing interest.

Ethical standards This article does not contain any studies with human participants or animals performed by any of the authors.

Open Access This article is distributed under the terms of the Creative Commons Attribution 4.0 International License (http:// creativecommons.org/licenses/by/4.0/), which permits unrestricted use, distribution, and reproduction in any medium, provided you give 
appropriate credit to the original author(s) and the source, provide a link to the Creative Commons license, and indicate if changes were made.

\section{References}

1. Adebahr S et al (2015) Lungtech, an eortc phase ii trial of stereotactic body radiotherapy for centrally located lung tumours: A clinical perspective. Br J Radiol 88:20150036

2. Al-Assar O et al (2014) Contextual regulation of pancreatic cancer stem cell phenotype and radioresistance by pancreatic stellate cells. Radiother Oncol 111:243-251

3. Bentzen SM (2006) Preventing or reducing late side effects of radiation therapy: Radiobiology meets molecular pathology. Nat Rev Cancer 6:702-713

4. Bhangu A et al (2014) The role of epithelial mesenchymal transition and resistance to neoadjuvant therapy in locally advanced rectal cancer. Colorectal Dis 16:O133-143

5. Brunner TB et al (2015) Sbrt in pancreatic cancer: What is the therapeutic window? Radiother Oncol 114:109-116

6. Busk M et al (2009) Can hypoxia-pet map hypoxic cell density heterogeneity accurately in an animal tumor model at a clinically obtainable image contrast? Radiother Oncol 92:429-436

7. Chang DT et al (2009) Stereotactic radiotherapy for unresectable adenocarcinoma of the pancreas. Cancer 115:665-672

8. Chetty IJ et al (2013) Correlation of dose computed using different algorithms with local control following stereotactic ablative radiotherapy (sabr)-based treatment of non-small-cell lung cancer. Radiother Oncol 109:498-504

9. Corradetti MN, Haas AR, Rengan R (2012) Central-airway necrosis after stereotactic body-radiation therapy. $\mathrm{N}$ Engl J Med 366:2327-2329

10. Duldulao MP et al (2013) Distribution of residual cancer cells in the bowel wall after neoadjuvant chemoradiation in patients with rectal cancer. Dis Colon Rectum 56:142-149

11. Fode MM, Hoyer M (2015) Survival and prognostic factors in 321 patients treated with stereotactic body radiotherapy for oligo-metastases. Radiother Oncol 114:155-160

12. Guckenberger M et al (2014) Definition of stereotactic body radiotherapy: Principles and practice for the treatment of stage i nonsmall cell lung cancer. Strahlenther Onkol 190:26-33

13. Guckenberger $\mathrm{M}$ et al (2016) Local tumor control probability modeling of primary and secondary lung tumors in stereotactic body radiotherapy. Radiother Oncol 118:485-491
14. Hoyer M et al (2005) Phase-ii study on stereotactic radiotherapy of locally advanced pancreatic carcinoma. Radiother Oncol 76:48-53

15. Kavanagh BD et al (2010) Radiation dose-volume effects in the stomach and small bowel. Int J Radiat Oncol Biol Phys 76:S101-S107

16. Kim DW et al (2014) Predictors of rectal tolerance observed in a dose-escalated phase 1-2 trial of stereotactic body radiation therapy for prostate cancer. Int J Radiat Oncol Biol Phys 89:509-517

17. Momm F et al (2010) Stereotactic fractionated radiotherapy for klatskin tumours. Radiother Oncol 95:99-102

18. Mothersill C, Seymour CB (2004) Radiation-induced bystander effects - implications for cancer. Nat Rev Cancer 4:158-164

19. Prokic V et al (2013) Whole brain irradiation with hippocampal sparing and dose escalation on multiple brain metastases: A planning study on treatment concepts. Int $\mathbf{J}$ Radiat Oncol Biol Phys $85: 264-270$

20. Schanne DH et al (2014) Stereotactic body radiotherapy for centrally located stage i nsclc : A multicenter analysis. Strahlenther Onkol 191(2):125-132

21. Sheehan JP et al (2014) Cranial stereotactic radiosurgery: Current status of the initial paradigm shifter. J Clin Oncol 32:2836-2846

22. Song SY et al (2009) Fractionated stereotactic body radiation therapy for medically inoperable stage i lung cancer adjacent to central large bronchus. Lung Cancer 66:89-93

23. Sterzing F et al (2014) Stereotactic body radiotherapy for liver tumors: Principles and practical guidelines of the degro working group on stereotactic radiotherapy. Strahlenther Onkol 190:872-881

24. Swaminath A, Dawson LA (2011) Image-guided radiotherapy strategies in upper gastrointestinal malignancies. Front Radiat Ther Oncol 43:315-330

25. Taylor ML, Kron T, Franich RD (2011) A contemporary review of stereotactic radiotherapy: Inherent dosimetric complexities and the potential for detriment. Acta Oncol (Madr) 50:483-508

26. Timmerman $\mathrm{R}$ et al (2006) Excessive toxicity when treating central tumors in a phase ii study of stereotactic body radiation therapy for medically inoperable early-stage lung cancer. J Clin Oncol 24:4833-4839

27. Timmerman RD (2008) An overview of hypofractionation and introduction to this issue of seminars in radiation oncology. Semin Radiat Oncol 18:215-222

28. van Baardwijk A et al (2012) Is high-dose stereotactic body radiotherapy (sbrt) for stage i non-small cell lung cancer (nsclc) overkill? A systematic review. Radiother Oncol 105:145-149

29. Withers HR, Taylor JM, Maciejewski B (1988) Treatment volume and tissue tolerance. Int J Radiat Oncol Biol Phys 14:751-759 